\title{
Epidemic hepatitis $C$ virus infection in Egypt: estimates of past incidence and future morbidity and mortality
}

\author{
E. M. Lehman ${ }^{1}$ and M. L. Wilson ${ }^{2}{ }^{1}$ International Epidemiology Institute, Rockville, MD; and ${ }^{2}$ Department of Epidemiology, \\ University of Michigan, Ann Arbor, MI, USA
}

Received October 2008; accepted for publication December 2008

SUMMARY. Hepatitis $\mathrm{C}$ virus (HCV) infection is gaining increasing attention as a global health crisis. Egypt reports the highest prevalence of $\mathrm{HCV}$ worldwide, ranging from $6 \%$ to more than $40 \%$ among regions and demographic groups. Predicting the impact of the epidemic has been difficult because of the long-latency period and low-resource setting. Accordingly, we sought to estimate historic incidence and predict the future impact of HCV using Markov simulation modelling techniques. Age-specific HCV incidence rates (IRs) were estimated using previously acquired age-specific HCV prevalence data. Data for this analysis were from a highly detailed, community-based seroprevalence study from 2003. Future HCV-related morbidity and mortality were estimated using a computer cohort simulation of HCV natural history in the Egyptian population. Population and natural history parameters were defined using results from a meta-analysis and existing comprehensive literature reviews. Incidence model estimates ranged from 2.01 to $25.47 \mathrm{HCV}$ cases per 1000 person-years (PYs). The highest IRs were calculated among those over 35 years of age. Our Markov model predicted 127821 deaths from chronic liver disease and 117556 deaths from hepatocellular carcinoma in Egypt over the next 20 years. During this period, it was estimated that HCV would yield $750210 \mathrm{PY}$ of decompensated cirrhosis, 132894 PY of hepatocellular carcinoma, and a total loss of 32.86 million years of life compared to a non-infected cohort. Our results support the claim of high HCV incidence in Egypt and suggest that HCV may lead to a substantial health and, consequently, economic burden over the next 10-20 years.

Keywords: developing countries, indirect incidence, infectious disease, liver cancer, Markov model.

\section{INTRODUCTION}

Hepatitis $\mathrm{C}$ virus (HCV) infection is gaining increasing attention as a global health problem, with approximately 3\% of the world's population infected [1,2]. These individuals are at greater risk for developing cirrhosis and/or hepatocellular carcinoma (HCC). Egypt reports the highest prevalence of $\mathrm{HCV}$ worldwide, ranging from $6 \%$ to more than $40 \%$ with an average of $13.8 \%$ [3-5]. In populations of blood transfusion recipients over the age of 30 , this proportion has been reported to be as high as $73 \%$, and in the general population aged $40-60$ years it can be as high as $55 \%$. At the same time, the prevalence in children is much lower, ranging between $2 \%$ and $10 \%$ [3-5]. While the levels in children are much lower than those seen in the older population, they are still excessive by World Health

Abbreviations: HCC, hepatocellular carcinoma; HCV, hepatitis C virus; HBV, hepatitis B virus; IR, incidence rate; PY, person-years; yo, years old.

Correspondence: Elizabeth M. Lehman, International Epidemiology Institute, 1455 Research Boulevard, Suite 550, Rockville, MD 20850, USA. E-mail: elizabeth@iei.ws
Organization (WHO) standards, where prevalences greater than about 3-4\% are considered high [2].

In Egypt, the major route of exposure to HCV appears to have been via widespread parenteral antischistosomal treatment (PAT), with more than 35 million injections given over a 20-year period (1960-1980) [3]. Despite termination of this program and the implementation of measures designed to reduce hospital-related infection, transmission continues.

Because the prevalence of $\mathrm{HCV}$ is exceeding that of $\mathrm{HBV}$ infection, HCV infection has become the leading risk factor for HCC in Egypt (antibodies present in as many as 75-90\% of HCC cases) [6]. The frequency of liver-related cancers (>95\% as HCC) relative to all cancers in Egypt has increased from approximately $4.0 \%$ in 1993 to $7.3 \%$ in 2003 [7]. Now the second highest cause of cancer incidence and mortality among men, HCC in Egypt occurs at rates significantly greater than those seen in surrounding Middle Eastern countries, as well as in the USA [8].

Incidence data are limited, however, because researchers have been plagued by logistical and methodological difficulties. Initial infection is rarely symptomatic, and chronic 
carriage is insidious. Thus, it is important to characterize the HCV situation in Egypt to predict the future health burden and guide appropriate health policy to confront the issue.

Various mathematical techniques provide practical approaches for making reasonable estimates about the history of the HCV epidemic and future predictions of HCVrelated morbidity and mortality. Methods for indirectly calculating age-specific incidence rates (IRs) using seroprevalence data were developed during the 1980s [9-11]. Saidel et al. [12] successfully validated one of these methods for HIV by comparing its predictions with observations from an incidence study. Such a method is advantageous, as it uses data from a single cross-sectional survey to estimate incidence and is particularly appropriate for developing countries where incidence data are frequently unavailable or unreliable.

The purpose of this study was to provide quantitative estimates of the burden of HCV infection among Egyptians. Burden was measured in two ways: First, we examined the burden of past infection incidence to further probe the hypothesis that the PAT campaign was largely responsible for the present epidemic in Egypt and shed light on the resulting epidemic curve, applying indirect estimates of historical IRs using seroprevalence data. Second, we used a Markov natural history model to estimate the impact of this epidemic, measured as future morbidity and mortality from sequelae of chronic HCV infection.

\section{MATERIAL AND METHODS}

\section{Prevalence and population data}

Age- and sex-specific population and mortality data for 5-year age groups were obtained from Egypt's Central Agency for Public Mobilization and Statistics (CAPMAS) and the WHO [13,14].

Prior systematic review of the literature identified high quality HCV prevalence studies for the period 1992-2005 [15]. Briefly, we selected five studies from the period 20012002, which we considered to have the greatest amount of detail and the highest methodological quality [4,16-19]. All studies were conducted on large $(n>1400)$ apparently healthy community-based populations. Third generation ELISA was used in all cases to test for HCV antibody. These studies covered ages 5-60+ years as well as geographic regions of interest (Upper and Lower Egypt). Since it is known that prevalence varies significantly between children and adults as well as between Upper and Lower Egypt, this sample of studies is highly representative of Egypt when considered in aggregate.

Age-specific prevalences across studies were compared using the chi-square test to determine if pooling results would be appropriate. No significant differences were found among adults from Lower Egypt, children from Lower Egypt, adults from Upper Egypt, or children from Upper Egypt. Summary age-specific prevalence measures, as well as male:female ratios from these studies, were calculated as weighted averages using a standard method [20]. The individual prevalence for each study was multiplied by the corresponding sample size and divided by the total (pooled) sample size of all studies. To populate the initial values of the HCV Infection health state in the Markov model, these summary age-specific prevalence proportions (adjusted for sex) were multiplied by their respective census population values.

\section{Incidence rate estimates}

The method for estimating age-specific IRs from prevalence data was originally described by Leske et al. [9], Podgor et al. [10] and Podgor and Leske [11]. Although initially developed for studying glaucoma, Saidel et al. [12] tested model predictions against observed incidence for HIV/AIDS and determined the model can provide crude estimates of 1-year age-specific IRs that are similar to those observed in longitudinal studies of HIV/AIDS. For these calculations, we chose to select only one of the studies mentioned above, so as not to introduce bias. Since we are inferring incidence measures, it is best not to pool studies where HCV seroprevalence was measured by different researchers on different populations. This decision was made in the interest of preserving internal validity at the expense of external validity and the ability to generalize results beyond the specific study population of interest. We chose to focus our investigation of incidence on the data from Arafa et al. [18], which presents age-specific prevalence from an area in Lower Egypt, the region known to have higher levels of HCV in Egypt [17].

Incidence rates are derived by using estimated prevalence of disease at the beginning and end of an age interval and the known age-specific mortality rates during the interval. The model is based on the functional relation between a risk (CI) and a rate (ID) as:

$$
\mathrm{CI}=1-\mathrm{e}^{-(\mathrm{ID} \times \Delta t)}
$$

where ID is incidence density, CI is cumulative incidence, and $\Delta t$ is the elapsed time (which could be defined by age differences), as long as the assumption of constant incidence within a time interval is not violated [12]. After accounting for age-specific mortality within age intervals, Leske et al. [9] propose the following:

$$
I_{x}=\frac{\ln \left(1-\Pi_{x}\right)}{\ln \left(1-q_{x}\right)+\ln \left(1-\Pi_{x}\right)} \quad \times \frac{1-\left(1-\Pi_{x}\right)\left(1-q_{x}\right)}{\left(1-1 / 2 q_{x}\right)}
$$

where $I_{x}$ is the incidence rate for the age interval; $q_{x}$ the probability of dying during the age interval; $x+1=$ age interval immediately following $x$

$$
\Pi_{x}=\frac{P_{x+1}-P_{x}}{1-P_{x}}
$$

where $P_{x}$ is the prevalence proportion at the beginning of the interval. 
After solving for $I_{x}$, it is divided by the number of years in the age interval to estimate the mean annual rate for time interval $x$. In 1983, Podgor et al. validated this model against other, more established methods [10]. For more details on the derivation and validation of this method, see Refs. [9-12]. Variance and standard error for model estimates were calculated using the 'delta method' described by Armitage and Leske [9,21], respectively, as:

$$
\operatorname{Var}\left(I_{x}\right)=\left[\partial I_{x} / \partial \Pi_{x}\right]^{2} \operatorname{Var}\left(\Pi_{x}\right)
$$

To use this model, it is necessary to address the following three assumptions:

1. Infection is lifelong and irreversible. We limit our analysis to chronically infected individuals, as the rate of remission is quite low. In addition, treatment for HCV in Egypt is limited and rarely successful in eliminating infection.

2. Homogeneity within age intervals. This model assumes mortality rates, disease IRs and population risk factors remain constant within the age intervals of analysis. We restricted age intervals to 5-year periods, making it reasonable to assume homogeneity of these characteristics within age categories.

3. Non-differential mortality rates between $\mathrm{HCV}+$ and $\mathrm{HCV}-$ individuals. Model estimates using this method are not robust when one age interval is followed by another with lower prevalence (results in a negative incidence) [12]. For the purpose of this analysis, therefore, age groups $>50$-years old (yo) were excluded, because prevalence levels begin to decline. Truncating this analysis to those $<50$ yo is reasonable, since the purpose is to identify recent incidence trends following the elimination of the PAT campaign, making younger age groups more relevant. Adjusting for differential mortality due to cirrhosis/ liver failure/HCC would be essential in populations $>50$ yo, but it is negligible in younger groups. Differential mortality is the result of long-term sequelae associated with chronic HCV infection. Previous work has shown that these sequelae have their greatest impact in the population over 50 yo and have little effect in younger individuals $[6-8,22]$. However, access to cirrhosis and other non-HCC liver disease mortality data was extremely limited despite extensive searching. In our own sensitivity analyses, we found the estimates to be fairly stable in the presence of varying mortality rates. Thus, because we limited age groups to $<50$ yo, no adjustment for differential mortality was needed.

\section{Future HCV-related morbidity/mortality}

Using Visual Basic for Microsoft Excel ${ }^{\mathrm{TM}}$, we developed a Markov simulation model to predict HCV-related morbidity and mortality among a cohort representative of the HCVinfected population in Egypt (Fig. 1; Table 1). Cohort mem-

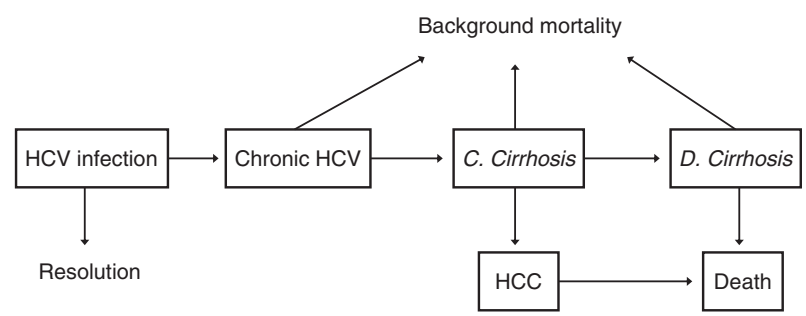

Fig. 1 Natural history model for Hepatitis C virus infection and its sequelae. Conceptual model showing the health states incorporated into the Markov simulation model. C, compensated; $\mathrm{D}=$ decompensated.

bers move among health states over 1-year time steps for 20 years. By tracking the proportion of the cohort developing sequelae, we were able to estimate the future health burden. The likelihood of health state transitions was based on probabilities derived from a review of the literature as well as publications from the WHO Hepatitis C Working Group (Table 1; the literature used to guide parameter estimates is cited in Appendix 1). The initial HCV Infection Health state was populated using the summary age-specific prevalence proportions discussed above and multiplying them by their respective census population estimates.

The model is set up to include ages 0-80 (time step $=1$ year). Anyone alive at age 80 years dies by age 81 . Since life expectancy in Egypt is 69 for males and 73 for females, and the population over 80 years of age accounts for only $0.3 \%$ of the total population, this age structure seems reasonable [14]. To incorporate aging into the model, we added the probability of remaining in a healthy state from one time step to the next, which is simply [1 - sum(transition probabilities to different health states)]. This exists for all health states except the original HCV infection. No one remains in the HCV infection health state after the first time step, when people are either moved to chronic infection, or removed from the system in the resolution health state.

Age- and sex-specific background mortality rates were obtained from the WHO and are applied to the following health states: chronic HCV infection, compensated cirrhosis, and decompensated cirrhosis [14]. All deaths from hepatocellular carcinoma are considered to be attributed to it. No deaths occur in the first time step. Typically, hepatitis $\mathrm{C}$ infection resolves or becomes chronic within the first year of infection [1,2]. Since our prevalence estimates are population-based, the first time step is designed to appropriately calculate the number of chronically infected individuals.

Sex is incorporated only insofar as generating the initial population structure and applying age-sex-specific background mortality. Although we hypothesize that transition probabilities may indeed be different by sex, they have yet to be clearly defined in the literature and from prospective cohort studies. Since previous research has indicated a 
Table 1 Health state transition probability estimates used in the Markov simulation

\begin{tabular}{|c|c|c|c|c|}
\hline Health state (i) & Health state $(i+1)$ & Annual probability & $\begin{array}{l}\text { Lower } \\
\text { bound }\end{array}$ & $\begin{array}{l}\text { Upper } \\
\text { bound }\end{array}$ \\
\hline \multirow[t]{2}{*}{ HCV infection } & Chronic infection & 0.75 & 0.50 & 0.85 \\
\hline & Infection resolution & 1-P(Chronic) & & \\
\hline \multirow[t]{2}{*}{ Chronic infection } & Compensated cirrhosis* & $0.05 / 20$ (<40 years $)$ & N/A & N/A \\
\hline & Background mortality $\dagger$ & $0.20 / 20(40+$ years $)$ & & \\
\hline \multirow[t]{3}{*}{ Compensated cirrhosis } & Decompensated cirrhosis & & & \\
\hline & Hepatocellular carcinoma & 0.065 & 0.04 & 0.092 \\
\hline & Background mortality $\dagger$ & 0.035 & 0.024 & 0.046 \\
\hline \multirow[t]{2}{*}{ Decompensated cirrhosis } & Death from D cirrhosis & & & \\
\hline & Background mortality $\dagger$ & 0.186 & 0.137 & 0.300 \\
\hline Hepatocellular carcinoma & Death from $\mathrm{HCC} \ddagger$ & 0.95 & & \\
\hline
\end{tabular}

Upper and lower bound estimates for sensitivity analysis were determined based on error associated with parameter estimates found in the literature.

*The compensated cirrhosis transition probability is 0.05 over 20 years if the individual was initially infected with HCV prior to 40 years of age. The transition probability is 0.2 over 20 years if the individual was aged 40 years or older at the time of initial HCV infection (Appendix 1). †Background mortality is defined by age-sex-specific rates obtained from WHO [14]. $₫$ All death from hepatocellular carcinoma is assumed to be attributed to it.

greater proportion of males are infected in Egypt vs females, sex is included to reflect these different initial values [37,16-19]. For the Markov model alone, it provides little beyond the initial values and the ability to project sex-specific health outcomes.

The disability or mortality burdens related to HCV infection were estimated. We used the computer simulation to determine the years of life spent by the cohort with decompensated cirrhosis or hepatocellular carcinoma (HCC). To estimate the years of life lost, we also compared life expectancy estimates for the chronic HCV population with those for an age- and sex-matched general population. These estimates calculated premature mortality and assumed permanent disability once patients developed decompensated cirrhosis or HCC, but did not consider quality-of-life deficits, as no such studies have been reported from Egypt that would provide appropriate quality of life information for HCV infection. The model was limited to 20 years because: (1) the natural history beyond 20 years is less certain, weakening our confidence in parameter estimates; and (2) estimates of disease and death are less meaningful, as they would not include incident cases who became infected during the period of analysis (due to the unavailability of incidence data).

\section{RESULTS}

\section{Age-specific prevalence}

All studies used in calculating summary measures had sample sizes greater than 1400 , reducing the probability of bias in prevalence estimates due to low power. Chi-square tests revealed no significant differences between studies measuring redundant populations (i.e. adults from Lower Egypt), so summary measures for the natural history model were calculated (not shown).

Summary prevalence estimates were calculated for the following age groups: 5-9, 10-14, 15-19, 20-29, 30-39, 40-49, and 50+. As expected, prevalence consistently increased with age except for a decrease between the age groups 40-49 and 50+. The decrease in the final age group could be related to lower past exposure to HCV through PAT which is tightly linked to HCV infection. Pooled estimates also revealed a male:female ratio of 1.2:1 for ages 0-19 years and 1.6:1 for individuals $\geq 20$.

\section{Incidence rate estimates}

Raw prevalence measures used in the calculation of IRs [18] were smoothed by fitting them to a logistic curve $\left(1 / \mathrm{e}^{(a-b x)}\right)$, where $a=-4.172$ and $b=-0.076$ (Fig. 2). Table 2 presents the results from the age-specific IR calculations. Mean annual IRs were obtained by dividing the model estimate by the number of years included in the age interval (5 years). Model estimates ranged from 2.01 to 25.47 per $1000 \mathrm{PY}$. The highest IRs were calculated for those $\geq 35$ yo. Such high incidence for these people is consistent with the age cohort effect seen with respect to the PAT campaign. We were unable to estimate the IRs for those $\geq 50$ yo, because prevalence significantly decreases beginning with the 55-59 yo age group. This particular method for estimating incidence loses accuracy under these conditions and frequently results in the calculation of a negative IR. 


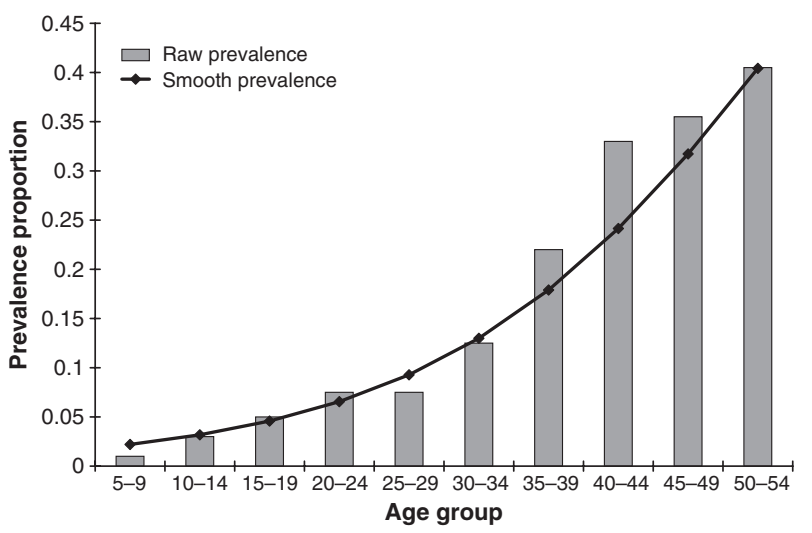

Fig. 2 Raw age-specific HCV prevalence proportions in Lower Egypt, 2002. Data from Arafa et al. [18] were used and plotted with smoothed age-specific prevalence estimates. Prevalence data were fit to a logistic curve: $1 / \mathrm{e}^{(-a-b x)}$ where $a=-4.172 ; b=-0.076$.

\section{Future HCV-related morbidity and mortality}

Predictions from the Markov model that estimate future HCV-related morbidity and mortality in Egypt, including the upper and lower bound estimates from sensitivity analysis, are striking (Table 3). Based on review of the prevalence studies, approximately 8.84 million individuals (4.98 million males and 3.86 million females) are estimated to be infected with HCV in Egypt, resulting in 6.62 million with chronic infection. Our model predicted that over the next 20 years a total of 127821 deaths due to decompensated cirrhosis would occur in this cohort, plus another 117556 deaths due to HCC. The highest proportion of deaths related to HCV will occur 10-20 years from now. Deaths due to HCC appear to peak within 15-16 years, whereas deaths due to decompensated cirrhosis only begin to flatten out in the final 2 years of analysis (Fig. 3). Deaths from HCC are greater than deaths from decompensated cirrhosis earlier in the model, due to the fact that individuals diagnosed with HCC in Egypt typically do not live past 1 or 2 years, whereas people with decompensated cirrhosis can live several years before succumbing to the condition.

In addition to the $244377 \mathrm{HCV}$-related deaths predicted by the model, when compared with an age-matched general population over the same time period, the HCV-infected cohort would have accumulated 3862643 years of compensated cirrhosis, 750210 years of decompensated cirrhosis, 132894 years of hepatocellular carcinoma, and the loss of 32.86 million years of life.

\section{DISCUSSION}

The objective of this study was to estimate past incidence of HCV using an indirect method and make projections about the future health burden using a natural history model. Despite Egypt's status as the country with the highest levels of HCV infection in the world, few studies have been done to characterize the dynamics of transmission, how they may have changed over time, and what this means for the future of health in Egypt. There is little doubt that the HCV epidemic is largely the result of some health care workers using unsterilized needles during the PAT campaign between the 1960s and the 1980s [3,6,23,24]. Beyond identifying PAT as the dominant source of infection, however, little research has been undertaken to characterize $\mathrm{HCV}$ dynamics and public health implications. Prevalence

\begin{tabular}{ccccc}
\hline $\begin{array}{l}\text { Age group } \\
\text { years })\end{array}$ & $\begin{array}{l}\text { Prevalence } \\
(\%)[\text { Raw }]\end{array}$ & $\begin{array}{l}\text { Prevalence } \\
(\%)[\text { Smooth] }\end{array}$ & Incidence (SE) & $\begin{array}{l}\text { Mean annual } \\
\text { incidence per } \\
1000 \text { PY }\end{array}$ \\
\hline $5-9$ & 1.0 & 2.2 & $0.010(0.01)$ & 2.01 \\
$10-14$ & 3.0 & 3.2 & $0.014(0.01)$ & 2.89 \\
$15-19$ & 5.0 & 4.6 & $0.021(0.01)$ & 4.13 \\
$20-24$ & 7.5 & 6.5 & $0.029(0.01)$ & 5.85 \\
$25-29$ & 7.5 & 9.3 & $0.041(0.03)$ & 8.19 \\
$30-34$ & 12.5 & 13.0 & $0.056(0.03)$ & 11.28 \\
$35-39$ & 22.0 & 17.9 & $0.076(0.04)$ & 15.21 \\
$40-44$ & 33.0 & 24.1 & $0.010(0.05)$ & 19.99 \\
$45-49$ & 35.5 & 31.7 & $0.127(0.06)$ & 25.47 \\
$50-54$ & 40.5 & 40.4 & - & - \\
$55-59^{*}$ & 32.0 & 49.8 & - & - \\
$60+*$ & 22.5 & 59.1 & - & - \\
\hline
\end{tabular}

Table 2. Indirectly estimated agespecific incidence rates for $\mathrm{HCV}$ in Lower Egypt

Incidence rates were calculated using smoothed age-specific prevalence measures. Data were fit to a logistic curve: $1 / \mathrm{e}^{(a-b x)}$ where $a=-4.172 ; b=-0.076 .{ }^{*}$ Not included in incidence calculations to preserve integrity of the smoothed prevalence estimates. 
Table 3 Predicted HCV-related morbidity and mortality

\begin{tabular}{lrrrr}
\hline Health state & Estimate & Lower bound & Upper bound \\
\hline Compensated cirrhosis (life years) & 3862643 & 2902917 & 3881476 \\
Decompensated cirrhosis (life years) & 750210 & 392042 & 818895 \\
Hepatocellular carcinoma (life years) & 132894 & 68132 & 176333 \\
Decompensated cirrhosis (deaths) & 127821 & 48728 & 227701 \\
Hepatocellular carcinoma (deaths) & 117556 & 59960 & 156713 \\
\hline
\end{tabular}

Estimates were measured as life years or number of deaths. Upper and lower bound estimates were obtained by varying parameter estimates in accordance with their margin of error as indicated by the literature.

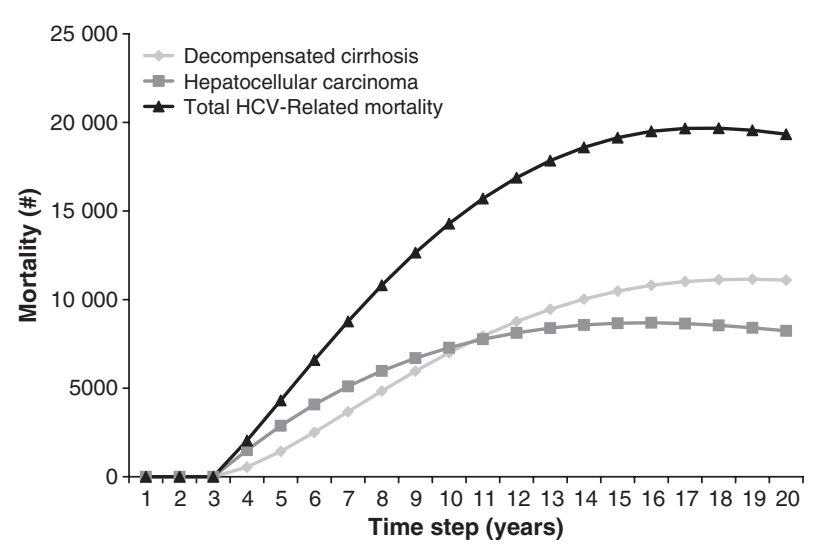

Fig. 3 Future HCV-related morbidity and mortality in Egypt. Predicted mortality due to HCV-related decompensated cirrhosis and hepatocellular carcinoma over a 20-year period.

studies show a significantly lower level of HCV infection among younger individuals, with rural populations approximately $12 \%$ and urban areas at only $2-3 \%[3,4,16-$ 19]. This still exceeds the WHO's definition of high endemicity (4\%), leaving Egypt's position as a leading country in the burden of HCV unchanged. Recent cross-sectional studies have found little evidence that formal medical procedures still contribute to transmission. In addition, informal medical procedures, such as circumcision performed by a barber, show significant associations but can only explain between $10 \%$ and $20 \%$ of the infections $[3,4,6]$. Some have suggested that intra-familial transmission may be crucial in transmission, but this hypothesis has yet to be specifically tested [25-27]. Although illuminating, such reports do not help with inferences regarding the future of HCV, and consequently the future of liver disease, in Egypt.

\section{Incidence estimates}

To address this knowledge gap, we calculated past age-specific HCV IRs using a method that analyses HCV prevalence data from a one-time, cross-sectional survey. Although originally developed for studying chronic diseases like glau- coma, Saidel et al. [12] demonstrated the validity of this method when compared with observational data from a HIV/AIDS study. As expected, we identified very high IRs among those $\geq 30$ yo. Among those $<30$ yo, largely comprised of people unaffected by the PAT campaign, we noted a continuous decline in IRs. Despite lower incidence among those 5-14 yo, IRs remained at 2-3 per 1000 PY, suggesting that transmission to children must still be occurring despite the elimination of PAT and intense measures to reduce other iatrogenic exposures. Future longitudinal studies are needed to gather observational data both to validate these findings as well as to test hypotheses regarding the current dominating modes of transmission.

We recognize that incidence estimates are only as valid as the model assumptions are robust. One important limitation of the model is the assumption of constant IRs [9-12]. The degree to which violation of this assumption may have affected model estimates is unpredictable and difficult to assess. We were unable to acquire prevalence data for age intervals of $<5$ years. Perhaps constancy was absent in some of these age groups, but the natural history of HCV, as well as the strong influence of widespread PAT and iatrogenic exposures, suggest that 5-year-age intervals were reasonable. Since the primary modes of transmission among younger people have yet to be clearly identified, the probability of violating the constant incidence assumption is likely greater, but 5-year intervals seem to be appropriate resolution for examining incidence among younger populations. Furthermore, because HCV has high prevalence in Egypt, the composition of risk groups is likely to change as the population at risk becomes saturated with infection. The steady-state condition assumed by the model may not have been fully met, suggesting that the relation between prevalence, incidence, and duration will only produce rough estimates [12].

The Podgor and Leske-based method can result in negative estimates of the IRs if prevalence at an older age is sharply less than at an earlier age [9,12]. Such estimates obviously are invalid, indicating either: (1) lack of power and/or too much variability among age groups, or (2) the model assumptions do not hold in the age range being examined. Therefore, we were unable to obtain incidence 
estimates for age groups $>50$ years when age-specific prevalence begins to decline. This decline among older age groups supports the hypothesis that the population aged 3050 years was the most affected by the PAT campaign. It would be valuable to gain prevalence data of the older population in smaller age group intervals and in different geographic locations to examine $\mathrm{HCV}$ incidence and PAT trends more closely.

Although the overall prevalence of HCV infection in Egypt is relatively high, the incidence of new infections needed to maintain this prevalence is still low enough that it is difficult to obtain precise incidence estimates. Therefore, results from our modelling approach should not be used to measure changes in IRs over time or differences in IRs between risk groups. Again, this was the principle reason why we chose to estimate incidence from only one study. While estimates from multiple studies could have been calculated, it would have been difficult to tease apart possible violations of assumptions when attempting to compare results or draw broader conclusions. True longitudinal incidence studies are still the best way to identify nuances in incidence trends across age, time and space.

\section{Future HCV-related morbidity and mortality in Egypt}

Our natural history model predicted 127821 deaths from decompensated cirrhosis and 117556 deaths from hepatocellular carcinoma due to the HCV epidemic over the next 10-20 years. We also calculated that the infected cohort would spend 750210 years in the diseased state of decompensated cirrhosis, 132894 years in hepatocellular carcinoma and lose 32.86 million years of life in comparison to an age-sex-matched non-infected cohort. These disturbing projections actually may have underestimated total HCVrelated morbidity and mortality for a few reasons. All cases of acute hepatitis were excluded, because Egypt does not have highly accurate data regarding acute infection and its natural history. Thus, we chose to limit our natural history model and projections to those with chronic HCV infection. In addition, the possibility of accelerated progression among older cases or those co-infected with HBV or HIV was not considered, cases that may be more likely to develop hepatic complications [28]. To date there has only been one other model constructed to look at HCV specifically in Egypt, and it involved fitting a model to historic data [29]. Although there was evidence that age- and sex-influenced parameter estimates, confirmatory observational data are not yet presently available for comparison. Consequently, we elected to work with a more conservative model, assuring a baseline, or underestimated, projection of future morbidity and mortality. We also did not evaluate HBV separately. Just as many people were infected with $\mathrm{HBV}$ as with $\mathrm{HCV}$ during the PAT campaign; however, HBV only caused chronic infections in $5 \%$ or less of infected individuals, whereas chronic HCV infection developed in $70-80 \%[3,6]$. This is related to the natural history of $\mathrm{HBV}$, where the probability of developing chronic infection decreases with age [30]. Since most people who received PAT were older than 10-15 years, they were at less risk for developing persistent HBV infection [6]. In addition, the $\mathrm{HBV}$ vaccine has been incorporated since 1992, which should lead to a steady decline in the significance of HBV infection, eventually playing only a minor role in the future of liver disease in Egypt.

Our analysis suggested the health burden related to premature mortality or disability from decompensated cirrhosis or hepatocellular carcinoma may be very high. The value of these estimates is that they inform policy makers about the rates at which age-specific groups are becoming infected with $\mathrm{HCV}$ and what these estimates will mean in terms of future morbidity and mortality. This information is crucial, because it can lead to conclusions about the level and direction of the HCV epidemic in Egypt that might be less readily apparent from the prevalence data alone. Despite the remarkable decline in new $\mathrm{HCV}$ infections since the end of the PAT campaign, mortality related to existing cases will likely continue to increase over the next 10-20 years, similar to the results of Deuffic-Burban et al. [29]. Hence, there is an urgent need to address this problem as HCV infection is frequently asymptomatic until cirrhosis develops, when treatment is less effective. More research regarding cost-effectiveness of screening for HCV infection and indications for treatment is needed to help guide public health policy in this area. Continued research on the natural history of HCV and the development of new treatments should remain top priorities not only for Egypt, but for global health as well.

\section{ACKNOWLEDGEMENTS}

The authors thank Professors R. Palmer Beasley, Hal Morgenstern, Susan Murray and Zhenhua Yang for their insightful comments and critical feedback during the development of this manuscript. E.M. Lehman was supported by a US National Science Foundation Graduate Research Fellowship and a Sigma Xi Grant-in-Aid of Research. Both E.M. Lehman and M.L. Wilson were supported on this project by the Global Health Program at the University of Michigan School of Public Health.

\section{REFERENCES}

1 Shepard CW, Finelli L, Alter MJ. Global epidemiology of hepatitis C virus infection. Lancet Infect Dis 2005; 5: $558-$ 567.

2 Wasley A, Alter MJ. Epidemiology of hepatitis C: geographic differences and temporal trends. Semin Liver Dis 2000; 20: $1-16$.

3 Frank C, Mohamed MK, Strickland GT et al. The role of parenteral antischistosomal therapy in the spread of hepatitis C virus in Egypt. Lancet 2000; 355: 887-891. 
4 El-Sadawy M, Ragab H, El-Toukhy H et al. Hepatitis C virus infection at Sharkia governorate, Egypt: seroprevalence and associated risk factors. J Egypt Soc Parasitol 2004; 34(1): 367-384.

5 Abdel-Aziz F, Habib M, Mohamed MK et al. Hepatitis C virus (HCV) infection in a community in the Nile Delta: population description and HCV prevalence. Hepatology 2000; 32: 111-115.

6 Strickland GT. Liver disease in Egypt: hepatitis C superseded schistosomiasis as a result of iatrogenic and biological factors. Hepatology 2006; 43: 915-922.

7 El-Zayadi AR, Badran HM, Barakat EM et al. Hepatocellular carcinoma in Egypt: a single center study over a decade. World J Gastroenterol 2005; 11: 5193-5198.

8 Freedman LS, Edwards BK, Ries LAG, Young JL, eds. Cancer Incidence in Four Member Countries (Cyprus, Egypt, Israel, and Jordan) of the Middle East Cancer Consortium (MECC) Compared with US SEER. Bethesda, MD: National Cancer Institute. NIH Pub. No. 06-5873, 2006.

9 Leske MC, Ederer F, Podgor MJ. Estimating incidence from age-specific prevalence in glaucoma. Am J Epidemiol 1981; 113(5): 606-613.

10 Podgor MJ, Leske MC, Ederer F. Incidence estimates for lens changes, macular changes, open-angle glaucoma and diabetic retinopathy. Am J Epidemiol 1983; 118(2): 206-212.

11 Podgor MJ, Leske MC. Estimating incidence from age-specific prevalence for irreversible diseases with differential mortality. Stat Med 1986; 5: 573-578.

12 Saidel T, Sokal D, Rice J, Buzingo T, Hassig S. Validation of a method to estimate age-specific Human Immunodeficiency virus (HIV) incidence rates in developing countries using population-based seroprevalence data. Am J Epidemiol 1996; 144(3): 214-223.

13 Central Agency for Public Mobilization and Statistics. Statistical Year Book, Cairo, Egypt, 2005.

14 World health statistics 2005. Indicators [online database]. Geneva: World Health Organization, 2005.

15 Lehman EM, Wilson ML. Epidemiology of hepatitis viruses among hepatocellular carcinoma cases and healthy people in Egypt: A systematic review and meta-analysis. Int $J$ Cancer 2009; 124(3): 690-697.

16 Mohamed MK, Magder LS, Abdel-Hamid M et al. Transmission of hepatitis $\mathrm{C}$ virus between parents and children. Am J Trop Med Hyg 2001; 75(1): 16-20.
17 Mohamed MK, Bakr I, El-Hoseiny M et al. HCV-related morbidity in a rural community of Egypt. J Med Virol 2006; 78: 1185-1189.

18 Arafa N, El Hoseiny M, Rekacewicz C et al. Changing pattern of hepatitis C virus spread in rural areas of Egypt. J Hepatol 2005; 43: 418-424.

19 Medhat M, Shehata M, Magder LS et al. Hepatitis C in a community in Upper Egypt: risk factors for infection. Am J Tropical Med Hyg 2002; 66(5): 633-638.

20 Reijnders J, Ehrt U, Weber WEJ, Aarsland D, Leentjens AFG. A systematic review of prevalence studies of depression in Parkinson's disease. Mov Disord 2007; 23(2): 183-189.

21 Armitage P. Statistical Methods in Medical Research. New York: John Wiley \& Sons, 1971.

22 Lehman EM, Soliman AS, Ismail K et al. Patterns of hepatocellular carcinoma incidence in Egypt from a population-based cancer registry. Hepatol Res 2008; 38: 465473.

23 El-Zayadi AR. Curse of schistosomiasis on Egyptian liver. World J Gastroenterol 2004; 10(8): 1079-1081.

24 Pybus OG, Drummond AJ, Nakario T, Robertson BH, Rambaut A. The epidemiology and iatrogenic transmission of hepatitis C virus in Egypt: a bayesian coalescent approach. Mol Biol Evol 2003; 20(3): 381-387.

25 Magder LS, Fix AD, Mikhail NN et al. Estimation of the risk of transmission of hepatitis $\mathrm{C}$ between spouses in Egypt based on seroprevalence data. Int J Epidemiol 2005; 34: 160-165.

26 Mohamed MK, Abdel-Hamid M, Mikhail NN et al. Intrafamilial transmission of hepatitis C in Egypt. Hepatology 2005; 42(3): 683-687.

27 Minola E, Baldo V, Baldovin T, Trivello R, Floreani A. Intrafamilial transmission of hepatitis C virus infection. Eur J Epidemiol 2006; 21(4): 293-297.

28 Global Burden of Hepatitis C Working Group. Global burden of disease (GBD) for hepatitis C. J Clin Pharmacol 2004; 44: 20-29.

29 Deuffic-Burban S, Mohamed MK, Larouze B, Carrat F, Valleron AJ. Expected increase in hepatitis C-related mortality in Egypt due to pre-2000 infections. J Hepatol 2006; 44: 455-461.

30 Yim HJ, Lok AS-F. Natural history of chronic hepatitis B virus infection: what we knew in 1981 and what we know in 2005. Hepatology 2006; 43(2 Suppl. 1): S173-S181.

\section{APPENDIX 1 LITERATURE INCLUDED IN THE META-ANALYSIS}

1 Afdhal NH. The natural history of hepatitis C. Semin Liver Dis 2004; 24(Suppl. 2): 3-8.

2. Alter HJ, Seeff LB. Recovery, persistence, and sequelae in hepatitis $\mathrm{C}$ virus infection: a perspective on long-term outcome. Sem Liver Dis 2000; 20(1): 17-35.

3. Bruno S, Silini E, Crosignani A et al. Hepatitis C virus genotypes and risk of hepatocellular carcinoma in cirrhosis: a prospective study. Hepatology 1997; 25: 754-758.

4. Chiaramonte M, Stroffolini T, Vian A et al. Rate of incidence of hepatocellular carcinoma in patients with compensated viral cirrhosis. Cancer 1999; 85: 21322137.

5. Degos F, Christidis C, Ganne-Carrie N et al. Hepatitis C virus related cirrhosis: time to occurrence of hepatocellular carcinoma and death. Gut 2000; 47: 131136.

6. Deuffic-Burban S, Mohamed MK, Larouze B, Carrat F, Valleron AJ. Expected increase in hepatitis C-related mortality in Egypt due to pre-2000 infections. J Hepatol 2006; 44: 455-461. 
7. Dore GJ, Freeman AJ, Law M, Kaldor JM. Natural history models for hepatitis C-related liver disease: different disease progression parameters for different settings. Antivir Ther 2003; 8(5): 365-372.

8. Fattovich G, Giustina G, Degos F et al. Morbidity and mortality in compensated cirrhosis type C: a retrospective follow-up study of 384 patients. Gastroenterology 1997; 112: 463-472.

9. Fattovich G, Pantalena M, Zagni I et al. Effect of hepatitis $\mathrm{B}$ and $\mathrm{C}$ infections on the natural history of compensated cirrhosis: a cohort study of 297 patients. Am J Gastroenterol 2002; 97: 2886-2895.

10. Gentilini P, Laffi G, La Villa G et al. Long course and prognostic factors of virus-induced cirrhosis of the liver. Am J Gastroenterol 1997; 92: 66-72.

11. Ginés P, Quintero E, Arroyo V et al. Compensated cirrhosis: natural history and prognostic factors. Hepatology 1987; 7: 122-128.

12. Global Burden of Hepatitis C Working Group. Global burden of disease (GBD) for hepatitis C. J Clin Pharmacol2004; 44: 20-29.

13. Goodgame B, Shaheen NJ, Galanko J, El-Serag HB. The risk of end stage liver disease and hepatocellular carcinoma among persons infected with hepatitis $C$ virus: publication bias? Am J Gastroenterol 2003; 98(11): 2535-2542.

14. Hu KQ, Tong MJ. The long-term outcomes of patients with compensated hepatitis $C$ virus-related cirrhosis and history of parenteral exposure in the United States. Hepatology 1999; 29: 1311-1316.
15. Imberti D, Fornari F, Sbolli G et al. Hepatocellular carcinoma in liver cirrhosis. A prospective study. Scand J Gastroenterol 1993; 28: 540-544.

16. Mandelli C, Fraquelli M, Fargion S et al. Comparable frequency of hepatocellular carcinoma in cirrhosis of different aetiology. Eur J Gastroenterol Hepatol 1994; 6: 1129-1134.

17. Serfaty L, Aumaitre H, Chazouilleres $\mathrm{O}$ et al. Determinants of outcome of compensated hepatitis C virus-related cirrhosis. Hepatology 1998; 27: 14351440.

18. Sypsa V, Touloumi G, Papatheodoridis GV et al. Future trends of HCV-related cirrhosis and hepatocellular carcinoma under the currently available treatments. J Viral Hepatol 2005; 12(5): 543-550.

19. Takano S, Yokosuka O, Imazeki F, Tagawa M, Omata M. Incidence of hepatocellular carcinoma in chronic hepatitis B and C: a prospective study of 251 patients. Hepatology 1995; 21: 650-655.

20. Tanaka J, Kumada H, Ikeda K et al. Natural histories of hepatitis $\mathrm{C}$ virus infection in men and women simulated by the Markov model. J Med Virol 2003; 70(3): 378386.

21. Thomas DL, Seeff LB. Natural history of hepatitis C. Clin Liver Dis 2005; 9(3): 383-398.

22. Tsai JF, Jeng JE, Ho MS, Chang WY, Hsieh MY, Lin ZY et al. Effect of hepatitis $\mathrm{C}$ and $\mathrm{B}$ virus infection on risk of hepatocellular carcinoma: a prospective study. $\mathrm{Br}$ J Cancer 1997; 76: 968-974. 